\title{
Structure of RNA-dependent RNA Polymerase 2 (RDR2) and its implications for double-stranded RNA synthesis in gene silencing \\ Yuichiro Takagi ${ }^{1}$, Akihito Fukudome ${ }^{2}$ \\ ${ }^{1}$ Biochemistry \& Molecular Biology, Indiana Univ School of Medicine ${ }^{2}$ Indiana University/HHMI ytakagi@iu.edu
}

RNA-dependent RNA polymerases (RdRP) play critical roles in RNA-mediated gene silencing. In Arabidopsis thaliana, RNA-Dependent RNA Polymerase 2 (RDR2) generates double-stranded RNAs (dsRNAs) from a single stranded RNA template generated by DNA-dependent nuclear RNA Polymerase IV (Pol IV). Resulting dsRNAs are then processed into short-interfering RNAs that guide RNA-directed DNA methylation and transcriptional gene silencing.

RDR2 is a single protein with a molecular mass of $130 \mathrm{kDa}$. In our ongoing attempt to elucidate how RDR2-Pol IV combination generates double-stranded RNAs (dsRNAs) from DNA templates, we determined the structure of RDR2 at $3.5 \AA$ A resolution by single-particle cryo-electron microscopy having overcome a preferred orientation issue utilizing the tilted datasets. The RDR2 structure does not exhibit the conserved architecture (fingers, palm, thumb) seen in the polymerase core of the viral RdRP family. Instead, structural features of RDR2 strikingly resemble to those of yeast DNA-dependent RNA polymerase II and bacterial RNA polymerase: such features include bridge helix, trigger loop and the well-conserved two double-psi-beta-barrel (DPBB) type catalytic core. N-terminus region (residues 1-100) contains a RNA-recognition motif (RRM) adjacent to a positively charged channel that extends to the catalytic center. The distance from the RRM to the active site corresponds to the length of a single-stranded RNA of $\sim 7 \mathrm{nt}$, which is consistent with our biochemical data that RNAs must be longer than $8 \mathrm{nt}$ to serve as templates for RDR2. In experiments mimicking the arrangement of DNA and RNA strands following Pol IV transcription, RDR2 will also engage unpaired RNA 3' ends generated upon re-annealing of the template and nontemplate DNA strands, with a requirement for $\sim 10 \mathrm{nt}$ of unpaired RNA. Collectively, our structural and biochemical data suggest a mechanism by which Pol IV arrest and backtracking causes its transcripts' 3 ' ends to become unpaired from the template DNA, allowing RDR2 to engage the nascent transcripts and extract them from Pol IV as a consequence of dsRNA synthesis. 